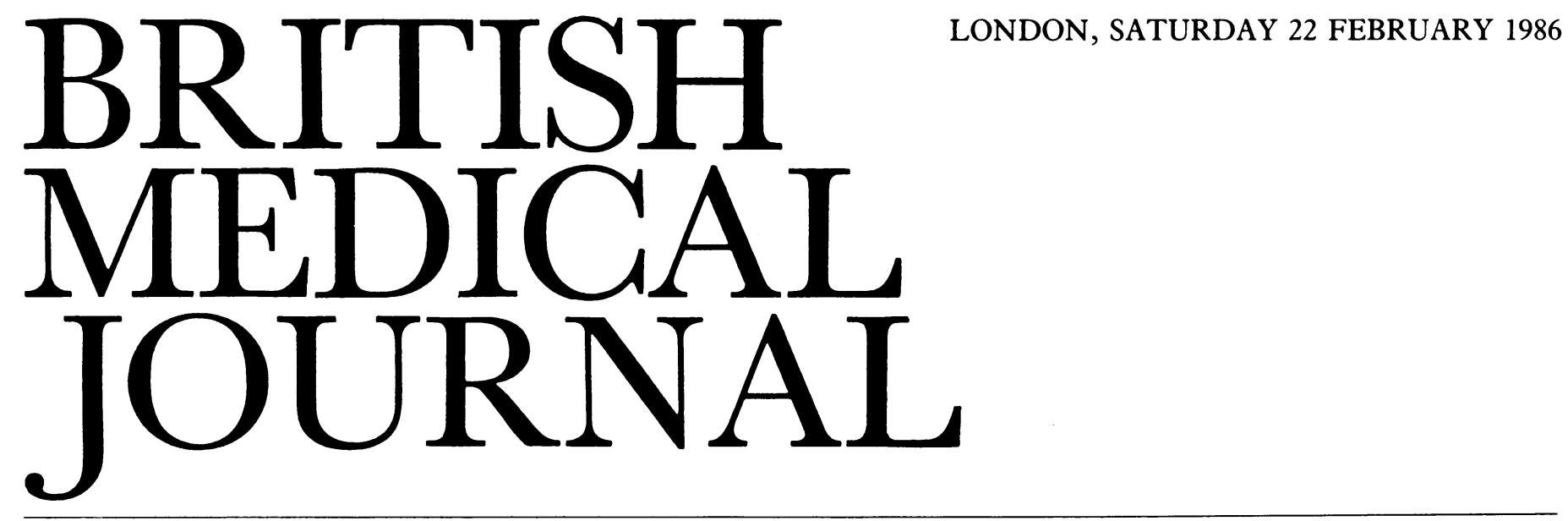

\title{
Screening for glaucoma
}

The glaucomas are a group of diseases sharing in common a characteristic appearance of the optic nerve head (glaucomatous cupping) and loss of the visual field of a "nerve fibre bundle" type. Four fifths of patients with glaucoma have an intraocular pressure exceeding the (statistically derived) upper limit of normal. About two thirds of glaucomas are primary; the remainder are secondary to other ocular disease. The glaucomas affect around one in 200 of the adult population. ${ }^{12}$ They are an important, common cause of blindness in adults. The prevalence rises with each decade; in our aging population the numbers affected by glaucomas are on the increase.

The primary adult glaucomas may be subdivided into two groups, open and closed angle glaucoma. In the former group the (usually) raised intraocular pressure is associated with obstruction in the one way valve system-the trabecular meshwork - to the exit of aqueous humour from the anterior chamber, while in the latter contact between the iris and the trabecular meshwork blocks its openings, rendering it ineffective.

The eye is an enclosed system with a high extravascular fluid pressure. This-the intraocular pressure-averages 15 $\mathrm{mm} \mathrm{Hg}$ with fluctuations throughout the day. The optic nerve may be damaged if the intraocular pressure becomes too high for tolerance. Usually this is the result of a longstanding rise in the intraocular pressure above "normal" intraocular pressure. ${ }^{3}$ In either case the result is deformation and eventual loss of the neurones passing from the retinal ganglion cells to the lateral geniculate body. ${ }^{4}$ The affected areas at the optic nerve head are similar in all patients with glaucoma, giving a characteristic pattern of loss of nerve fibres and so "typical" glaucomatous defects in the visual field.

If the intraocular pressure is lowered the rate of progression of visual loss may be retarded if not halted altogether ${ }^{5}$; and in theory the glaucomas are an eminently treatable cause of visual loss.

Acute angle closure glaucoma and secondary glaucomas are usually identified early in the disease. By contrast, the commoner primary chronic open or closed angle glaucomas tend to be insidious in onset and slowly progressive. These are asymptomatic in the early stages, and patients tend to present late in the disease. As these diseases are easier to treat in the early stages considerable thought has gone into methods of identifying them in the presymptomatic phase.

Screening for glaucoma requires ways of identifying the cardinal signs noted above: rise in the intraocular pressure, glaucomatous cupping, and defects in the visual field. Testing of visual fields plays a small part: it is time consuming, and the inaccuracies seen in the patient's response the first time his fields are tested often mask early defects. Those patients with extensive visual loss and therefore identifiable visual field defects will have concomitant changes at the optic nerve head which may be identified by direct ophthalmoscopy. Alternative tests of visual function using retinal contrast sensitivity, hue discrimination, and pattern electroretinography have shown early promise, but they have not been shown to be sensitive or specific enough to be used as screening tests.

Measurement of the intraocular pressure is simple and rapid. Patients with pressures above "normal" are readily identified. The intraocular pressure has a linear relationship with systolic blood pressure, ${ }^{6}$ while the incidence of glaucoma is high in diabetics. ${ }^{7}$ Unfortunately, the identification of "raised" intraocular pressure does not mean that the patient has glaucoma-nor is it an indication that he will develop glaucoma. Furthermore, some individuals with glaucoma do not have raised intraocular pressure. ${ }^{12}$ Nevertheless, about one in 10 patients found to have ocular hypertension will have glaucoma at the time of screening, ${ }^{8}$ and $10 \%$ of the remainder will develop glaucoma within 10 years. ${ }^{9}$ The risk of developing glaucoma rises with the intraocular pressure ( $2 \%$ in 10 years in patients with an intraocular pressure of less than $28 \mathrm{~mm} \mathrm{Hg}$ rising to $45 \%$ of those with an intraocular pressure over $35 \mathrm{~mm} \mathrm{Hg}$ ). ${ }^{9}{ }^{10}$ Patients with ocular hypertension should be considered at risk and kept under observation. If the risk is thought high enough for visual loss to be certain in five years or so then prophylactic treatment should be given.

Considerable attention has been given to the changes in the appearance of the optic disc in patients with glaucoma. Loss in the visual field may be suspected when the depletion of neurones at the rim of the optic disc results in enlargement of the orifice of the optic cup. ${ }^{11}$ The normal optic cup (orifice) in whites occupies up to three to four tenths of a horizontal or vertical diameter; it is wider in West Indians and some patients with myopia. The two cups in the eyes of one patient are of similar size and shape. Diffuse loss of retinal neurones occurring in some early glaucomas causes a concentric enlargement of the cup, which will usually differ in size from its mate. This occurs before detectable visual field loss. ${ }^{12}$ Focal loss of neurones is seen with the "nerve fibre bundle" 
type of field defects and gives rise to localised thinning or absence of the neuroretinal rim.

Though this focal change may also be seen in a few patients with an ischaemic optic neuropathy and in patients with pits and colobomas of the optic disc, the progressive changes that occur in glaucoma allow the correct diagnosis to be made. Such changes may be seen on direct ophthalmoscopic examination. Firstly, the optic disc should be examined, checking its size and the thickness of the neuroretinal rim. ${ }^{.1}$ Secondly, concomitant alterations should be sought in the course of the surface blood vessels on the optic disc (described as "baring of the circumlinear vessel"'s and "kinking" of the surface vessels $\left.{ }^{14}\right)$; these identify the site of an acquired change secondary to enlargement of the optic cup. Next, the peripapillary retinal nerve fibre layer should be examined for defects or "grooves." 15 16 Finally, ophthalmological examination may show a shallow anterior chamber; all patients with an axial anterior chamber depth of less than $2.5 \mathrm{~mm}$ despite a normal intraocular pressure should undergo indentation gonioscopy to check for peripheral anterior synechiae in the upper half of the angle. Patients with synechiae may be suffering from progressive angle closure leading to chronic angle closure glaucoma. They need to be kept under observation and in some cases considered for a prophylactic iridotomy.

Who should screen for glaucoma? At present most new referrals of patients with glaucoma come through general practitioners from opticians. ${ }^{17}$ Usually the opticians have suspected glaucoma from the results of tonometry augmented by ophthalmoscopy and perimetry. Such screening is patchy, because opticians tend to see two select population groups: firstly, those with symptoms from presbyopia-who being in the 40s and 50s are below the peak age level for the onset of chronic glaucoma - and, secondly, those with visual symptoms from eye disease which, if from glaucoma, will reflect an advanced stage. Furthermore, not all opticians carry out this type of screening.

General practitioners are better placed to identify asymptomatic patients with glaucoma. They usually see most of the elderly patients under their care, especially those with the risk factors of systemic hypertension and diabetes. With sufficient skill in the use of the direct ophthalmoscope a general practitioner could identify most early glaucomas developing in his patients. ${ }^{18} 19$ In reality if he is to recognise the changes occurring in the optic disc a general practitioner would need to have had far more teaching in direct ophthalmoscopy than that given to the average medical student. If ophthalmic outpatient departments are not to be swamped with patients with "query glaucoma" the referring ophthalmoscopist must have a developed ability to recognise these physical signs. We should perhaps note that in recent years the greatest efforts at nationwide tuition of general practitioners in the recognition of glaucoma have been funded by a pharmaceutical company making drugs for treating the disease.

Screening need not be restricted to individuals but may be extended to groups of patients. Indeed, since the prevalence of glaucomas in the adult population is only $0.5 \%$ unselected population screening is not cost effective. ${ }^{19} 20$ Screening of the optic discs may be part of a mass screening programme, however, and the identification of abnormalities may be made fairly mechanical. ${ }^{21}{ }^{22}$ Screening of individuals at high risk is considerably more cost effective. On occasion, glaucoma runs in families, ${ }^{23}$ and this has encouraged screening of close family members of known glaucoma sufferers ${ }^{24}$ In a retent review of 1700 such relatives, the incidence of overt glaucoma at the time of initial screening was $2 \%$, while $20 \%$ 무 had physical signs putting them at risk of developing the 3 disease. ${ }^{25}$

Long term follow up of such patients has enabled some $c$ weighting to be given to the risk factors. The main ones are a positive family history, an intraocular pressure exceeding 28 $\mathrm{mm} \mathrm{Hg}$, a cup:disc ratio exceeding $0 \cdot 6$, and increasing $\underset{\infty}{\mathbb{D}}$ age. ${ }^{26}{ }^{27}$ Less important factors include myopia, diabetes, or systemic hypertension, a hypertensive response to ocular $\stackrel{9}{.}$ steroids, and a shallow anterior chamber..$^{26}$ At present $\overrightarrow{\vec{F}}$ prophylactic hypotensive treatment is given to patients with $\stackrel{0}{0}$ ocular hypertension, particularly if other risk factors coexist. 들 All such patients need to be followed indefinitely, albeit $\frac{\vec{\sigma}}{\bar{c}}$ infrequently, and their numbers will steadily increase- $-\widehat{\Phi}$ which should mean an expansion of the existing glaucoma o services.

Eight years ago a leader writer on screening of glaucoma outlined the problems in its identification. ${ }^{28}$ Since then follow $\overrightarrow{\vec{\omega}}$ up of suspects has shown how screening may be refined. A combination of history taking and ophthalmoscopy should 3 identify most of the asymptomatic patients at risk. Sub- î sequent ophthalmological checks will then sort out those ? requiring treatment for established disease, prophylactic of treatment, follow up on suspicion, or reassurance and $\vec{\varphi}$ discharge.

Consultant Ophthalmic Surgeon,

ROGER A HITCHINGs

Moorfields Eye Hospital, London WC1V 2AN

1 Hollows FC, Graham PA. Intraocular pressure, glaucoma and glaucoma suspects in a defined population. Brf Ophthalmol 1966;50:570-86.

2 Kini MM, Leibowitz AM, Colton T, Nickerson RJ, Ganley J, Dawber TB. Prevalence of senile cataract diabetic retinopathy, senile macular degeneration and open angle glaucoma in the Framingham eye study. Am $\mathcal{f}$ Ophthalmol 1978;85:28-34.

3 Quigley HA, Addicks EM, Green WR. Optic nerve damage in human glaucoma. Arch Ophthalmol 1982;100:135-46.

4 Emery JM, Landis D, Paton D, Boniuk M, Craig JM. The laminar cribrosa in normal and glaucomatous human eyes. Transactions of the American Academy of Ophthalmology and Otolaryngology 1974;78:290-7. 5 Grant GW, Burke JF. Why do some people go blind from glaucoma? Ophthalmology 1982;89:
991-8.

6 Bengtsson B. The prevalence of glaucoma. Br f Ophthalmol 1981;65:46-9.

Kahn HA, Leibowitz AM, Gangley JP, et al. The Framingham eye study. 2. Association of ophthalmic pathology with single variable previously measured in the Framingham study. $A m$ 으 I Epidemiol 1977;106:33-8.

8 Eddy DM, Sanders LE, Eddy F. The value of screening for glaucoma with tonometry. Surv Ophthalmol 1983;28:194-205.

9 Kitazawa Y, Horie T, Aokis S, Susuki M, Nisnioka K. Untreated ocular hypertension. A long term prospective study. Arch Ophthalmol 1977;95:1180-4.

10 David R, Livingtone DG, Luntz MH. Ocular hypertension-a long term follow-up of treated and untreated patients. Br $\mathcal{f}$ Ophthalmol 1977;61:668-74.

11 Airaksinen PJ, Drance SM, Douglas GR, Schulzer M. Neuroretinal rim areas and visual field indices in glaucoma. Am $\mathcal{f}$ Ophthalmol 1985;99:107-10.

12 Pederson JE, Anderson DR. The mode of progressive disc cupping in ocular hypertension and glaucoma. Arch Ophthalmol 1980;98:490-5.

13 Herschler S, Oshler RH. Baring of the circumlinear vessel. An early sign of optic nerve damage. Arch Ophthalmol 1980;98:865-9.

14 Sharma NK, Hitchings RA. A comparison of monocular and "stereoscopic" photographs of the optic disc in the identification of glaucomatous visual field defects. $\mathrm{Br} \mathcal{f}$ Ophthalmol 1983;67:677-80.

15 Sommer A, Quigley HA, Robin AL, Miller NR, Ktaz J, Arkell MS. Evaluation of the nerve fibre layer assessment. Arch Ophthalmol 1984;102:1766-71.

16 Airaksinen PJ, Drance SM, Douglas GR, Schultzer M, Wijsman K. Visual field and retinal nerve fibre layer comparisons in glaucoma. Arch Ophthalmol 1985;103:205-7.

17 MacKean JM, Elkington AR. Referral routes to hospital of patients with chronic open-angle glaucoma. Br Med f 1982;285:1093-5.

18 Bengtsson B. Manifest glaucoma in the aged (2) cases detected by ophthalmoscopy. Acta Ophthalmol (Copenh) 1981;59:332-5.

19 Levi L, Schwartz B. Glaucoma screening in the health care setting. Surv Ophthalmol 1983;28: Gottlieb

Ophthalmol 1983;28:206-26.
Optien

21 Shiose $\mathrm{Y}$, Komuro K, Itoh T, Amano M, Kawase $\mathrm{Y}$. A new system for mass screening of glaucoma as part of autom multiphasic health screening services. Jap 7 Ophthalmol 1981:25:160-77.

22 Hitchings RA, Brown DB, Anderton SA. Glaucoma screening by means of an optic disc grid. $\mathrm{Br} f$ Ophthalmol 1983;67:352-5.

23 Paterson G. Studies of siblings of patients with both angle-closure and chronic simple glaucoma Trans Ophthalmol Soc UK 1961;81:561-76.

$24 \mathrm{MacKean}$ JM, Elkington AR. Alerting close relatives of patients with glaucoma. Br Med $\mathrm{J}$

1984;289:800-1.
25 Hitchings RA, Wooding S, Powell D, Coakes RL. Glaucoma family screening. Symposium on $\frac{\varrho}{\sigma}$ the detection of early glaucoma. Rome 1984 (in press).

26 Hart WM, Yablonski ME, Kass MA, Becker B. Multivariate analysis of the risk of glaucomatous visual field loss. Arch Ophthalmol 1979;97:1455-8.

27 Yablonski ME, Zimmerman TJ, Kass MA, Becker B. Diagnostic significance of optic disc cupping in ocular hypertensive patients. Am f Ophthalmol 1980;89:585-92.

28 Anonymous. Screening for glaucoma (Editorial). Lancet 1977;ii:437-8. 